\title{
PRÓTESE DE TRANSIÇÃO X PRÓTESE SOBRE IMPLANTES: RELATO DE CASO
}

\author{
TRANSITIONAL PROSTHESIS X IMPLANT-SUPPORTED PROSTHESIS: CASE REPORT
}

\section{Ana Beatriz Vilela TEIXEIRA'; Mariana Lima da Costa VALENTE²; Denise Tornavoi de CASTRO³; Victor Garone MORELLI"; Andréa Cândido dos REIS ${ }^{5}$}

1 - Mestre em Reabilitação Oral, Aluna de Doutorado em Reabilitação Oral. Departamento de Materiais Dentários e Prótese, Faculdade de Odontologia de Ribeirão Preto - Universidade de São Paulo.

2 - Doutorada em Reabilitação Oral. Departamento de Materiais Dentários e Prótese, Faculdade de Odontologia de Ribeirão Preto - Universidade de São Paulo.

3 - Doutorada em Reabilitação Oral. Departamento de Materiais Dentários e Prótese, Faculdade de Odontologia de Ribeirão Preto - Universidade de São Paulo.

4 - Mestre em Reabilitação Oral. Departamento de Materiais Dentários e Prótese, Faculdade de Odontologia de Ribeirão Preto - Universidade de São Paulo, Ribeirão Preto.

5 - Professora Associada do Departamento de Materiais Dentários e Prótese, Faculdade de Odontologia de Ribeirão Preto - Universidade de São Paulo.

\section{RESUMO}

Objetivo: O objetivo desse trabalho foi descrever um caso clínico, realizado na Faculdade de Odontologia de Ribeirão Preto - Universidade de São Paulo, com adaptação da prótese parcial removível do paciente em prótese total provisória de transição, após a instalação de implantes odontológicos. Material e método: O paciente era usuário de prótese parcial removível associada à prótese parcial fixa inferior, cujos pilares eram os elementos 42 e 44 . Ambos os dentes apresentavam indicação para extração. Após a remoção da prótese fixa e extração dos dentes 42 e 44, foram instalados quatro implantes na região anterior da mandíbula, e confeccionada uma prótese total de transição, a partir da prótese parcial removível do paciente. Após o período de osseointegração, a prótese sobre implantes definitiva foi confeccionada. Conclusão: A adaptação de prótese parcial removível em prótese total provisória de transição reduz o tempo dos procedimentos clínicos e o custo do tratamento, permitindo ao paciente a manutenção de suas funções orais, até a confecção da prótese definitiva.

PALAVRAS-CHAVE: Reabilitação bucal; Implantes dentários; Prótese temporária.

\section{INTRODUÇÃO}

Implantes osseointegráveis são uma opção de tratamento de elevado sucesso para ausências dentais, e ampliam as possibilidades de reabilitações protéticas, inclusive em relação à estética e requisitos funcionais ${ }^{1,2}$. Entretanto, em alguns casos, após a instalação dos mesmos, é requerido um período de 3 a 6 meses para a osseointegração antes da reabilitação final ${ }^{3-5}$.

O surgimento dos protocolos de carga imediata se deu através do conhecimento da biologia dos tecidos e o desenvolvimento de novos implantes, que permitiram o rápido restabelecimento funcional e estético do paciente. Entretanto, nos casos em que a estabilidade primária não é satisfatória, a aplicação de carga imediata está contraindicada, uma vez que são produzidos micro movimentos que induzem a formação de tecido fibroso, comprometendo o processo de osseointegração $\mathrm{O}^{1,6,7}$.

A transição dos pacientes dentados para desdentados gera prejuízo à função mastigatória, fonética, estética e até alterações psicológicas, o que diminui o sucesso e a colaboração do paciente com o tratamento ${ }^{8,9}$. Para evitar tais transtornos, é importante a confecção de próteses provisórias de transição logo após o procedimento cirúrgico ${ }^{10}$.
O conceito de prótese de transição e as diversas técnicas de confecção da mesma vêm sendo publicadas desde $1914^{11}$, esse tipo de prótese apresenta caráter provisório e é confeccionada a partir da prótese que o paciente já faz uso. Um exemplo é a adaptação de uma prótese parcial removível já existente em prótese total, com acréscimo de dentes artificiais e uma base em resina acrílica na região dos dentes extraídos ${ }^{12}$.

A adaptação de uma prótese parcial removível pode ser viável quanto ao tempo, permitindo maior flexibilidade no tratamento ${ }^{13}$, o cirurgião-dentista faz as alterações na prótese do paciente em poucas horas, transformando-a em prótese total de transição, de forma versátil e simples, facilmente incorporada à prática clínica.

A utilização de próteses de transição, além de fazer a proteção da ferida cirúrgica e auxiliar na osseointegração, evita a recessão gengival, perda óssea e permite a manutenção dessas áreas antes da colocação de uma prótese definitiva, diminuindo assim, complicações de tecidos moles como a peri-implantite e a profundidade vestibular inadequada, que podem ocorrer nas regiões dos implantes osseointegrados ${ }^{11,14-16}$.

Esse trabalho descreve um caso clínico, realizado na Faculdade de Odontologia de Ribeirão Preto - Universidade de São Paulo, 
com adaptação de uma prótese parcial removível do paciente em uma prótese total provisória de transição, após a instalação de implantes odontológicos, com o objetivo de reduzir o tempo dos procedimentos clínicos e o custo do tratamento, além de permitir a manutenção, funções orais e estética do paciente, até a confecção da prótese definitiva.

\section{RELATO DE CASO}

Paciente do sexo feminino, 75 anos, compareceu à clínica da Faculdade de Odontologia de Ribeirão Preto - Universidade de São Paulo, fazendo uso de uma prótese parcial removível inferior associada à uma prótese parcial fixa de três elementos $(42,43$ e 44) por meio de attachments (Figura 1), e uma prótese total no arco superior. A queixa principal da paciente era a instabilidade das próteses.

Na anamnese constatou-se ausência de sintomas relativos à disfunção na articulação temporomandibular e doenças sistêmicas. Após a realização do exame intraoral e radiografia panorâmica, indicou-se a exodontia dos elementos 42 e 44, seguido de instalação de implantes na região anterior da mandíbula e indicação de prótese Overdenture implantossuportada, devido à falta de volume ósseo para instalação de implantes na região posterior da mandíbula.

Para otimização dos custos e tempo clínico, na mesma sessão foi realizada a remoção da prótese parcial fixa, exodontia dos elementos 42 e 44, e instalação de quatro implantes, com dimensões de $\varnothing 3,5$ x 10 mm, do tipo Cone Morse (Alvim CM, Neodent ${ }^{\circledR}$, Curitiba, PR, Brasil), na região dos elementos 31, 33, 41 e 43. Após abertura do retalho, foi realizada a fresagem do nicho ósseo com as brocas na sequência indicada pelo fabricante, seguido da instalação dos implantes e sutura após o término do procedimento cirúrgico (Figura 2).

Antes dos procedimentos cirúrgicos, deu-se início a adaptação da prótese parcial removível da paciente em prótese total de transição. Para esse procedimento, foi confeccionada uma muralha de silicona de condensação pesada (Speedex, Vigodent ${ }^{\odot}$ Coltene S.A. Indústria e Comércio, Rio de Janeiro, RJ, Brasil) para moldar a prótese parcial fixa e a prótese parcial removível, diretamente na cavidade bucal. A impressão em negativo do espaço da prótese parcial fixa (Figura 3) foi preenchido com resina acrílica autopolimerizável, na cor dos dentes artificiais (Dencôr ${ }^{\circledR}$, Clássico Artigos Odontológicos, Campo Limpo Paulista, SP, Brasil), pela Técnica de Nealon ${ }^{17}$. Para a confecção da prótese total de transição, foi realizado o preparo da prótese parcial removível com fresa, para tornar a superfície áspera e melhorar a adesão da nova base. Após a presa da resina acrílica (Dencôr ${ }^{\circledR}$ ), foi adaptada uma cera utilidade no interior da prótese para simular o rebordo, e em seguida obteve-se a porção gengival com resina acrílica autopolimerizável rosa (Clássico ${ }^{\circledR}$, Clássico Artigos Odontológicos, Campo Limpo Paulista, SP, Brasil).

Finalizada a prótese total de transição, realizou-se a prova clínica, os ajustes, reembasamento com condicionador de tecido (Soft Confort $^{\circledR}$, Dencril, Pirassununga, SP, Brasil), acabamento e polimento, instalação e orientações ao paciente (Figura 4). Após o período de 4 meses para a osseointegração, confeccionou-se a prótese overdenture sobre implantes definitiva (Figuras 5 e 6). Foi realizado acompanhamento do caso clínico durante 1 ano, e observado que a prótese continua em perfeitas condições estéticas e funcionais.

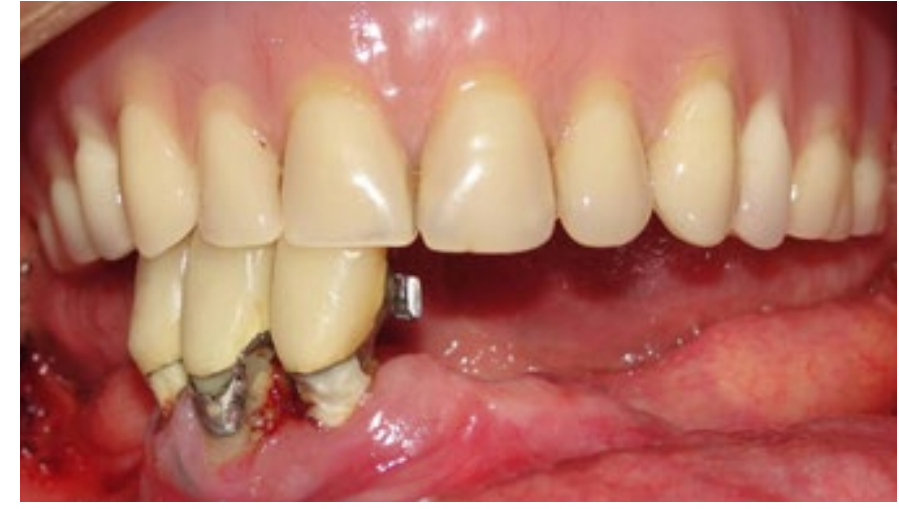

Figura 1 - Caso clínico inicial, com prótese parcial fixa inferior de 3 elementos

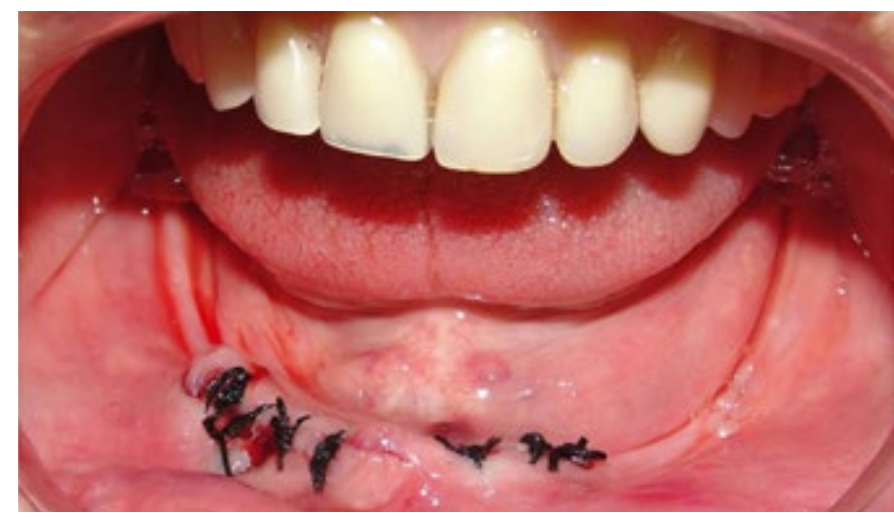

Figura 2 - Sutura do rebordo inferior após a instalação de quatro implantes

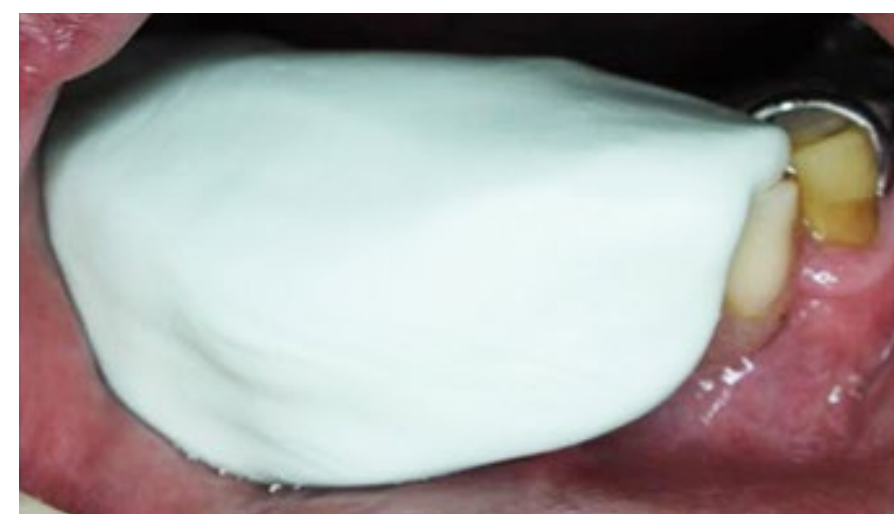

Figura 3 - Molde da prótese parcial fixa associada a prótese parcial removível inferior

A descrição desse caso clínico, bem como sua aprovação para publicação estão registradas no Comitê de Ética em Pesquisa da Faculdade de Odontologia de Ribeirão Preto ( $\mathrm{n}^{\mathrm{o}} 2008.1 .1259 .58 .9$ ).

\section{DISCUSSÃO}

A reabilitação oral com próteses implantossuportadas, quando bem planejada, apresenta elevado índice de sucesso ${ }^{4,18}$. Assim, é imprescindível a manutenção dos tecidos gengivais, evitando sobrecarga sobre os implantes, e a promoção da estabilidade das estruturas musculares, articulações e dimensões específicas que garantem a reabilitação oral ${ }^{4}$.

A prótese provisória que o paciente fará uso representa uma das mais importantes etapas do planejamento, podendo 
ser ela fixa ou removível ${ }^{4}$, visto que esta definirá as necessidades estéticas, funcionais e adaptativas do paciente, reduzindo a possibilidades de falhas e iatrogenias do ponto de vista estético-funcional.

A prótese provisória também pode ser utilizada como um guia em áreas com menor qualidade óssea, para garantir a longevidade do implante e detectar o seu mau posicionamento durante o ato cirúrgico. Além disso, também permite a obtenção de uma previsibilidade da osseointegração, antes da instalação da prótese definitiva ${ }^{19,20}$. Uma vez que, o planejamento adequado da prótese de transição evita a má distribuição de tensões no osso que circunda o implante, causando reabsorção óssea e microfissuras, que podem provocar a falha do mesmo ${ }^{21,22}$.

A prótese provisória total imediata é uma alternativa para casos em que não é recomendada a aplicação de carga nos implantes logo após a cirurgia. Esse tipo de prótese auxilia no processo de adaptação do paciente da condição de dentado para desdentado. Segundo o Glossário de termos protéticos, a prótese total imediata é definida como uma prótese total ou parcial removível, confeccionada para ser instalada imediatamente após a extração dos dentes naturais ${ }^{12}$.

De Rouck et al. ${ }^{23}$ (2009), em estudo comparando tecidos moles em implantes com instalação de coroa provisória com carga imediata e prótese parcial removível com carregamento tardio, relataram que implantes colocados imediatamente após as extrações dentárias nem sempre podem receber carga imediata, devido à falta de estabilidade primária. E observaram que a sobrevida do implante, a remodelação óssea, a profundidade de sondagem e o sangramento gengival não foram influenciados pelas formas de reabilitação desenvolvidas no estudo.

A confecção de uma prótese provisória de transição, a partir de uma prótese já existente, apresenta vantagens como: otimização do tempo clínico, redução dos custos, conforto ao paciente quanto à estética, função, fonética, proteção da ferida cirúrgica, controle da hemorragia, rapidez na cicatrização e facilidade na higienização; além de rápido retorno do paciente às suas atividades sociais e transição menos perceptível da condição dental, garantindo seu bem-estar psicológico e social ${ }^{13,14}$.

Além disso, a prótese de transição possibilita o restabelecimento da arquitetura gengival desejada, por meio do condicionamento tecidual ${ }^{24}$, e da anatomia dos tecidos moles, favorecendo a cicatrização dos mesmos, além da obtenção de uma mucosa de melhor qualidade, fator importante para a realização da prótese definitiva ${ }^{25,26}$. Por meio da instalação prévia desse tipo de prótese é possível prever a dimensão vertical de oclusão, estética e funcionalidade ideais para serem reproduzidas na prótese definitiva, garantindo o sucesso do tratamento.

Dessa forma, a transformação de uma prótese que o paciente faz uso, em uma prótese total de transição, é eficaz quando o cirurgião-dentista determina que a aplicação de carga imediata ao implante não é indicada devido às limitações cirúrgicas, estabilidade primária insatisfatória, necessidade de procedimentos cirúrgicos adicionais ou tempo de cicatrização prolongada ${ }^{23}$.

O tema abordado retrata a necessidade da observação de algumas características da prótese antiga utilizada pelo paciente com a finalidade de restabelecer provisoriamente a condição bucal do paciente com facilidade, tempo clínico e custos reduzidos.

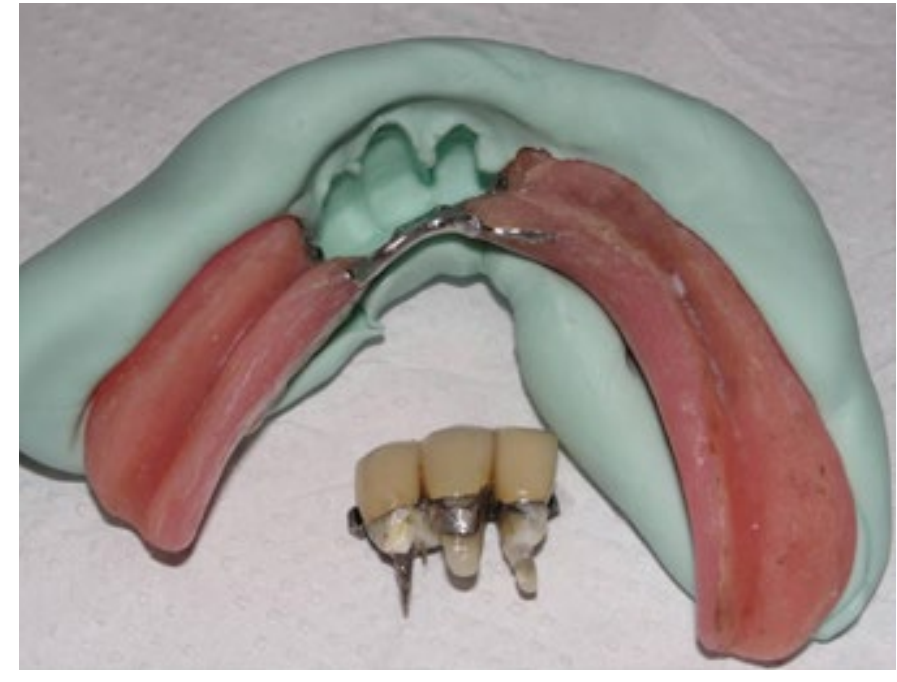

Figura 4 - Instalação da prótese total provisória de transição

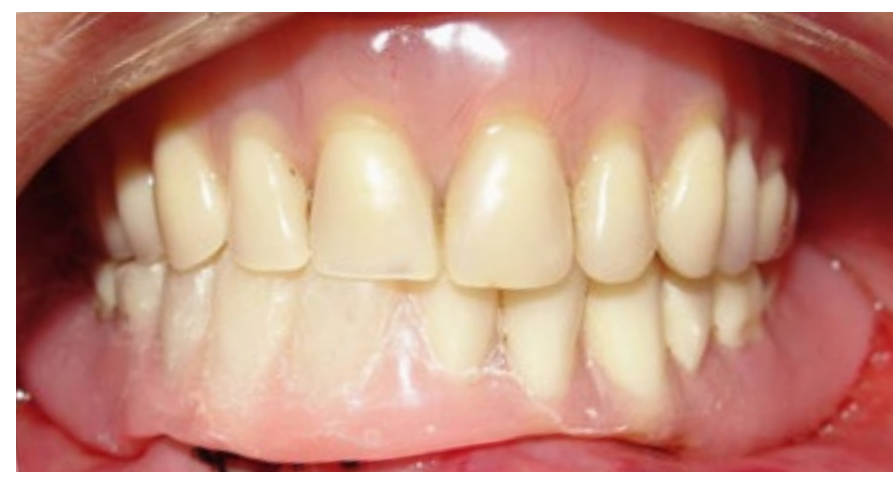

Figura 5 - Componentes protéticos instalados aos implantes

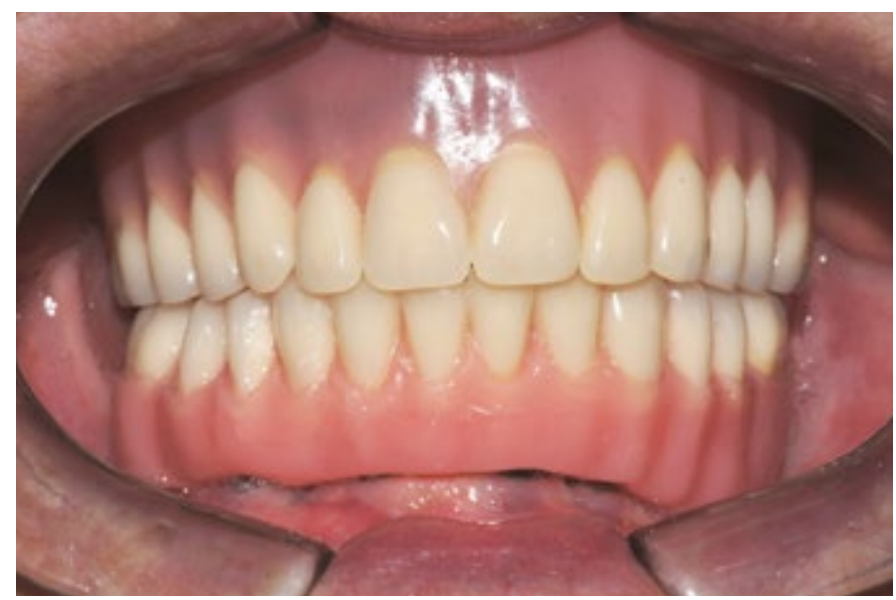

Figura 6 - Prótese overdenture definitiva instalada

\section{CONCLUSÃO}

Com base no planejamento proposto pode-se concluir que o uso de prótese provisória de transição auxilia na saúde e bem-estar do paciente, na preservação dos tecidos remanescentes, favorecendo também o cirurgião-dentista com a diminuição do tempo dos procedimentos clínicos, otimizando custos e garantindo a devolução e preservação das funções mastigatórias, fonéticas e restabelecimento da estética. 


\section{REFERÊNCIAS}

01. Valente MLC, Castro DT, Shimano A, Lepri CP, Reis AC. Analyzing the influence of a new dental implant design on primary stability. Clin Implant Dent Relat Res. 2016; 18(1): 168-73.

02. Reis AC, Paula WN, Marcantonio Jr E, Boeck Neto RJ, Rovere Neto DD, Mazzo CR. Influência do formato do implante para reabilitação de casos com limitação de espaço mesiodistal. Rev Fac Odontol Univ Passo Fundo. 2010; 15(3): 314-8.

03. Vasilios C, kourtis S, Katsikeris N, Nagy W. Tooth- and tissuesupported provisional restorations for the treatment of patients with extended edentulous spans. J Esthet Restor Dent. 2009; 21(1): 7-17.

04. Cho SC, Shetty S, Froum S, Elian N, Tarnow D. Fixed and removable provisional options for patients undergoing implant treatment. Comp Cont Educ Dent. 2007; 28(11): 604-8.

05. Kwon KR, Sachdeo A, Weber HP. Achieving immediate function with provisional prostheses after implant placement: A clinical report. J Prosthet Dent. 2005; 93(6): 514-7.

06. Bilhan H, Geckili O, Mumcu E, Bozdag E, Sünbüloğlu E, Kutay O. Influence of surgical technique, implant shape and diameter on the primary stability in cancellous bone. J Oral Rehabil. 2010;37(12): 900-7.

07. Esposito M, Grusovin MG, Maghaireh H, Worthington HV. Interventions for replacing missing teeth: different times for loading dental implants. Cochrane Database Syst Rev. 2013; 5(3): 1-94.

08. Goiato MC, Torcato LB, Santos DM, Moreno A, Antenucci RM, Carvalho Dekon SF. Quality of life and satisfaction of patients wearing implant-supported fixed partial denture: a cross-sectional survey of patients from Araçatuba city, Brazil. Clin Oral Implants Res. 2015; 26(6): 701-8.

09. Vieira RA, Melo AC, Budel LA, Gama JC, Mattias Sartori IA, Thomé G. Benefits of rehabilitation with implants in masticatory function: is patient perception of change in accordance with the real improvement? J Oral Implantol. 2014; 40(3): 263-9.

10. Papaspyridakos $\mathrm{P}$, Chronopoulos V. Transition from failing dentition to complete-arch implant rehabilitation with a staged approach: A 3-year clinical report. J Prosthet Dent. 2014; 112(3): 423-8.

11. Payne SH. A Transitional Dentadure. J Prosthet Dent. 1964; 14(2): 221-30.

12. The glossary of prosthodontic terms. The academy of prosthodontics. J Prosthet Dent. 2005; 94(1): 1-83.

13. Misch CM. Immediate loading of definitive implants in the edentulous mandible using a fixed provisional prosthesis: The denture conversion technique. J Oral Maxillofac Surg. 2004; 62(9): 106-15.
14. Sisson J, Boberick K, Winkler S. Conversion of a removable partial denture to a transitional complete denture: A clinical report. J Prosthet Dent. 2005; 93(5): 416-8.

15. Park JB. Increasing the width of keratinized mucosa around endosseous implant using acellular dermalmatrix allograft. Implant Dent. 2006; 15(3): 275-81.

16. Caneva M, Botticelli D, Viganò P, Morelli F, Rea M, Lang NP. Connective tissue grafts in conjunction with implants installed immediately into extraction sockets. An experimental study in dogs. Clin Oral Implants Res. 2013; 24(1): 50-6.

17. Nealon FH. Acrylic restorations by the operative nonpressure procedure. J Prosth Dent. 1952; 2(4):513-527

18. Giordano M, Ausiello P, Martorelli M. Accuracy evaluation of surgical guides in implant dentistry by non-contact reverse engineering techniques. Dent Mater. 2012; 28(9): e178-85.

19. Buser D, Von Arx T. Surgical procedures in partially edentulous patients with ITI implants. Clin Oral Implants Res. 2000; 11(1): 83-100.

20. Buser D, Martin W, Belser UC. Optimizing esthetics for implant restorations in the anterior maxilla: anatomic and surgical considerations. Int J Oral Maxillofac Implants. 2004; 19(supl. 19): 43-61.

21. Rocha EP, Kina S, Almeida EO, Carvalho NA, Freitas Jr AC, Anchieta RB. Short implant to support maxillary restorations: bone stress analysis using regular and switching platform. J Craniofac Surg. 2012; 23(3): 678-81.

22. Pjetursson BE, Lang NP. Prosthetic treatment planning on the basis of scientific evidence. J Oral Rehabil. 2008; 35(supl. 1): 72-9.

23. De Rouck T, Collys K, Wyn I, Cosyn J. Instant provisionalization of immediate single-tooth implants is essential to optimize esthetic treatment outcome. Clin Oral Impl Res. 2009; 20(6): 566-70.

24. El Askary AS. Use of connective tissue grafts to enhance the esthetic outcome of implant treatment: A clinical report of 2 patients. J Prosthet Dent. 2002; 87(2): 129-32.

25. Hilgenberg SP, Orellana-Jimenez EE, Sepúlveda-Navarro WF, Arana-Correa BE, Alves DCT, Campanha NH. Evaluation of Surface Physical Properties of Acrylic Resins for Provisional Prosthesis. Mat Res. 2008; 11(3): 257-60.

26. Palacci P, Nowzari H. Soft tissue enhancement around dental implants. J Periodontol. 2008; 47: 113-32.

\section{ABSTRACT}

Objective: The aim of this study was to describe a case, performed at the Dental School of Ribeirão Preto - University of São Paulo, with adaptation of the removable partial denture of the patient in a transitional denture after the installation of dental implants. Material and Methods: Patient was a user of a mandibular removable partial denture associated with fixed partial dentures, whose pillars were teeth 42 and 44 , both indicated for extraction. After removal of the fixed partial dentures and extraction of 42 and 44 , four implants were installed in the anterior region of the mandible, and a transitional denture was made from the patient's removable partial denture. After the period of osseointegration, the definitive implant prosthesis was made. Conclusions: The adaptation of a removable partial denture in a transitional denture reduces the time of the clinical procedures and the cost of the treatment, allowing to the patient to maintain his/her oral functions, until the definitive implant prosthesis is made.

KEYWORDS: Mouth rehabilitation; Dental implants; Denture temporary. 


\section{AUTOR PARA CORRESPONDÊNCIA}

Profa. Dra. Andréa Candido dos Reis

Departamento de Materiais Dentários e Prótese

Faculdade de Odontologia de Ribeirão Preto - Universidade de São Paulo

Avenida do Café, s/n, CEP: 14040-904, Ribeirão Preto, São

Paulo, Brasil

Tel: +55-16-3315-0477

E-mail: andreare@forp.usp.br 